\title{
Morphological and Molecular Characterization of Toxocara tanuki (Nematoda: Ascaridae) from Korean Raccoon Dog, Nyctereutes procyonoides koreensis
}

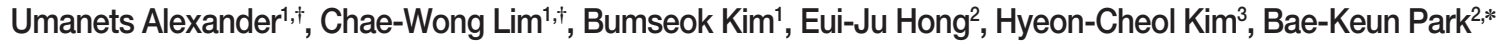 \\ ${ }^{1}$ College of Veterinary Medicine, Chonbuk National University, Iksan 54596, Korea; ${ }^{2}$ College of Veterinary Medicine, Chungnam National University, \\ Daejeon 34134, Korea; ${ }^{3} \mathrm{College}$ of Veterinary Medicine, Kangwon National University, Chuncheon 24341, Korea
}

\begin{abstract}
Present study was performed to describe the morphological and molecular characterization of Toxocara tanuki (Nematoda: Ascaridae) from Korean raccoon dog, Nyctereutes procyonoides koreensis, naturally infected in the Republic of Korea (Korea). Juvenile and adult worms of $T$. tanuki were recovered in 5 out of 10 raccoon dogs examined and the larval worms were detected in 15 out of 20 muscle samples (75\%). Small lateral alae were observed on the cranial end of the body in male and female adults and 2 long spicules (3.0-3.5 mm) were characteristically observed in the posterior end of males. In SEM observation, 18 pairs of proximal precloacal, a precloacal median, a postcloacal median and 5 pairs of postcloacal papillae were uniquely revealed in the posterior portion of males, but the proximal papillae were not shown in the lateral ends of females. Molecular analysis on the 18S rRNA partial DNA sequences was revealed the same finding in both samples, adult worms and muscle larvae, which are closely related to T. tanuki. In conclusion, it was confirmed for the first time that $T$. tanuki is indigenously distributed, the Korean raccoon dog is acted as the natural definitive host of this nematode in Korea and the morphological characteristics of $T$. tanuki were shown in specific structure for single postcloacal median papilla in male.
\end{abstract}

Key words: Nyctereutes procyonoides koreensis, Toxocara tanuki, morphological and molecular characterization, larva migrans, Korean raccoon dog, Korea

\section{INTRODUCTION}

The Korean raccoon dog (Nyctereutes procyonoides koreensis) is one of the most common mammals among canid wildlifes in the ecosystems of the Republic of Korea [1]. And some Korean workers have investigated (or surveyed) the gastrointestinal parasites in this species of mammals. Shin et al. [2] detected 4 species of helminth eggs (Toxocara sp., Trichuris sp., Capillaria sp., and hookworm eggs) and a species of protozoan cyst, Giardia sp. by fecal examination. Furthermore, Shin et al. [3] reported that raccoon dogs are infected with Pygidiopsis summa, Spirometra erinacei, Toxocara sp., hookworm, and a certain species of echinostome. Eo et al. [4] extensively examined total 882 fecal samples of wild raccoon dogs and they detected the ascarid eggs from $50 \%$ samples.

\footnotetext{
- Received 2 April 2018, revised 4 October 2018, accepted 27 October 2018.

*Corresponding author (bkpark@cnu.ac.kr)

† These authors contributed equally to this work.

(C) 2018, Korean Society for Parasitology and Tropical Medicine

This is an Open Access article distributed under the terms of the Creative Commons Attribution Non-Commercial License (http://creativecommons.org/licenses/by-nc/4.0) which permits unrestricted non-commercial use, distribution, and reproduction in any medium, provided the original work is properly cited.
}

Toxocara tanuki is a very common ascarid nematode in raccoon dogs in Japan, but only a few reports on it are available even in Japan $[5,6]$. Additionally, the ascarid species from raccoon dogs has not been determined in the 3 previous studies performed in Korea [2-4]. Therefore, we are going to identify the ascarid nematode from Korean raccoon dog using scanning electron microscopy (SEM) and DNA analysis in this study.

\section{MATERIALS AND METHODS}

Host animal, the Korean raccoon dog, examined

Twenty raccoon dogs from Jeollabuk-do (Province), Korea were used in this study. Raccoon dogs were obtained from the Wildlife Rescue and Conservation Center at Chonbuk National University, between February 2012 and May 2013. Some raccoon dogs were found dead, while others had died after rescue because they were in a severe stage of canine distemper.

At our diagnostic laboratory, necropsies were completed using a routine protocol. Skin was completely removed before necropsy to prevent sample contamination. 


\section{Sample collection}

All muscles sample and parasites were harvested during the necropsy of dead animals. Adult parasites were collected from the intestines. The lateral and cranial ends of the intestines were fixed with forceps to prevent their contents from leaking out. After they were opened, we performed a gross examination of the intestines for parasites.

We used the artificial digestion method to isolate larvae from the muscles [7]. Muscle samples (each $50 \mathrm{~g}$ ) from the neck, head, forelimb, and hindlimb were collected from all raccoon dogs $(n=20)$, and necropsy was performed on 10 of 20 raccoon dogs.

We isolated parasite eggs from fecal material, which was collected during necropsy. Sheather's sugar centrifugal flotation technique was used for egg detection [8]. A superficial liquid layer was observed by light microscopy.

\section{Morphological examination}

Adult parasites were collected from the intestines after necropsy. The worms were placed in lacto-phenol solution $(20 \mathrm{ml}$ glycerin, $10 \mathrm{ml}$ lactic acid, $10 \mathrm{~g}$ phenol, and $10 \mathrm{ml}$ distilled water) for $24 \mathrm{hr}$ until they became transparent.

For the SEM, the worms were washed 5 times with $0.2 \mathrm{M}$ cacodylate buffer ( $\mathrm{pH} 7.3$ ), fixed in $2.5 \%$ glutaraldehyde, and post-fixed in $1 \%$ osmium tetroxide at $4^{\circ} \mathrm{C}$. We then dehydrated the specimen in a graded ethyl alcohol series, dried using the $\mathrm{CO}_{2}$ critical point dryer (CPD 030, BAL-TEC), coated with gold, and then examined with the SEM (S-4800, Hitachi) at $15 \mathrm{kV}$.

\section{DNA extraction and amplification}

We prepared the parasite samples for DNA extraction following a previous protocol [9]. Parasite larvae and adult worm were washed 5-10 times in PBS. Two $\mu \mathrm{l}$ of Tris-HCl and one drop of mineral oil were added into $5 \mu \mathrm{l}$ of PBS containing larvae. Samples were heated at $90^{\circ} \mathrm{C}$ for $10 \mathrm{~min}$, and then cooled on ice. After adding $3 \mu \mathrm{l}$ of proteinase K solution (20 $\mathrm{mg} / \mathrm{ml}$ ), samples were incubated overnight at $56^{\circ} \mathrm{C}$. To inactivate the proteinase $\mathrm{K}$, parasite samples were heated at $90^{\circ} \mathrm{C}$ for $10 \mathrm{~min}$, and then stored at $-20^{\circ} \mathrm{C}$ until use.

We extracted genomic DNA from the parasite samples using the GeneAll Exgene ${ }^{\mathrm{TM}}$ Tissue SV mini kit according to the manufacturer's instructions. We aligned several sequences from $T$. tanuki, Toxocara canis, and Toxocara cati by multiple sequence alignment (Clustal Omega, http://www.ebi.ac.uk/Tools/msa/ clustalo/), and then common primers for Toxocara were de- signed using an online tool (Primer3input, http://primer3. ut.ee/). An $18 \mathrm{~S}$ rRNA region spanning the internal transcribed spacer 1 (ITS1) gene was amplified by PCR. The oligonucleotide sequences of primers employed to detect the T. tanuki $18 \mathrm{~S}$ rRNA region spanning the ITS1 gene (DNA) were 5'-AAAGTCTCCGAATGTGCATAAG-3' (forward) and 5'-TGCATTGACAGCTCTCCTCA-3' (reverse). The primer sets were designed for a 398 bp product. PCR was performed with standard conditions: $95^{\circ} \mathrm{C}$ for $30 \mathrm{sec}$, annealing at $60^{\circ} \mathrm{C}$ for $30 \mathrm{sec}$, and extension at $72^{\circ} \mathrm{C}$ for $1 \mathrm{~min}$. The PCR reaction was performed in a Takara PCR Thermal Cycler (Takara, Shiga, Japan) using EmeraldAmp GT PCR Master Mix (Takara) with $1 \mu \mathrm{l}$ of the DNA aliquot. The PCR products were visualized on a $1.5 \%$ agarose gel, and then purified using QIAquick PCR purification kit (Qiagen, Alameda, California, USA).

\section{Sequence analysis and phylogenetic analysis}

For the PCR sequence analysis, amplifications were directly sequenced using the ABI Prism Big Dye terminator v. 3.0 ready reaction cycle sequencing kits (Applied Biosystems, Foster City, California, USA) with the same primers as those used in PCR. The sequencing reactions were performed on a PE Applied Biosystems 3100 automated sequencer. Approximately $120 \mathrm{bp}$ of the $5^{\prime}$ and $3^{\prime}$ ends of the sequencing data were trimmed because of non-specific sequencing due to by primer binding, and the trimmed sequence revealed a PCR products that were 282-398 bp. The sequence data were aligned on the Clustal Omega program (clustal O 1.2.1). A phylogenetic tree based on the sequence analysis was generated by Neighbor-Joining method using Blast tree (http://blast.ncbi.nlm.nih.gov/Blast.cgi).

\section{RESULTS}

\section{Infection status with ascarids in Korean raccoon dogs}

The insensitivity of the infestation ranged 0.02-1.3 larvae/g, and the maximum number of the larva harvest was 65 larvae in $50 \mathrm{~g}$ muscle sample (Table 1).

Juvenile and adult worms of T. tanuki were observed in the jejunum, ileum, and colon. Approximately 1-19 worms (mean 2.8) were counted per animal. The intestinal nematodes were found in 5 of 10 animals (50\%).

\section{Morphological characteristics of adult worm}

In males, the body of adults measured 42.5-45 mm (mean $43.8 \mathrm{~mm}$ ) in length and 0.9-1.0 mm in width $(\mathrm{n}=10)$. In fe- 
Table 1. Prevalence of the intestinal nematode and muscular larvae in raccoon dogs

\begin{tabular}{cccccc}
\hline $\begin{array}{c}\text { No. } \\
\text { dog }\end{array}$ & $\begin{array}{c}\text { Amount of } \\
\text { muscle } \\
\text { tissue (g) }\end{array}$ & $\begin{array}{c}\text { No. of } \\
\text { larva }\end{array}$ & Larva/g & $\begin{array}{c}\text { Adult } \\
\text { nematode in } \\
\text { intestine }\end{array}$ & $\begin{array}{c}\text { Fecal egg } \\
\text { examination }\end{array}$ \\
\hline 1 & 50 & 3 & 0.06 & $\mathrm{NE}^{\mathrm{a}}$ & $\mathrm{NE}$ \\
2 & 50 & 7 & 0.14 & $\mathrm{NE}$ & $\mathrm{NE}$ \\
3 & 50 & 0 & - & $\mathrm{NE}$ & $\mathrm{NE}$ \\
4 & 30 & 11 & 0.36 & $\mathrm{NE}$ & $\mathrm{NE}$ \\
\hline 5 & 50 & 65 & 1.30 & $\mathrm{NE}$ & $\mathrm{NE}$ \\
6 & 50 & 3 & 0.06 & $\mathrm{NE}$ & $\mathrm{NE}$ \\
\hline 7 & 50 & 1 & 0.02 & $\mathrm{NE}$ & $\mathrm{NE}$ \\
8 & 50 & 0 & - & $\mathrm{NE}$ & $\mathrm{NE}$ \\
\hline 9 & 15 & 1 & 0.70 & $\mathrm{NE}$ & $\mathrm{NE}$ \\
10 & 15 & 0 & - & $\mathrm{NE}$ & $\mathrm{NE}$ \\
11 & 20 & 3 & 0.15 & 3 & + \\
12 & 20 & 1 & 0.05 & 19 & + \\
13 & 20 & 0 & - & 0 & - \\
14 & 20 & 3 & 0.15 & 1 & - \\
15 & 20 & 1 & 0.05 & 2 & + \\
16 & 20 & 1 & 0.05 & 0 & - \\
17 & 20 & 2 & 0.10 & 3 & - \\
18 & 20 & 0 & - & 0 & - \\
19 & 20 & 2 & 0.10 & 0 & - \\
20 & 20 & 3 & 0.15 & 0 & - \\
\hline
\end{tabular}

NE, Not examined.
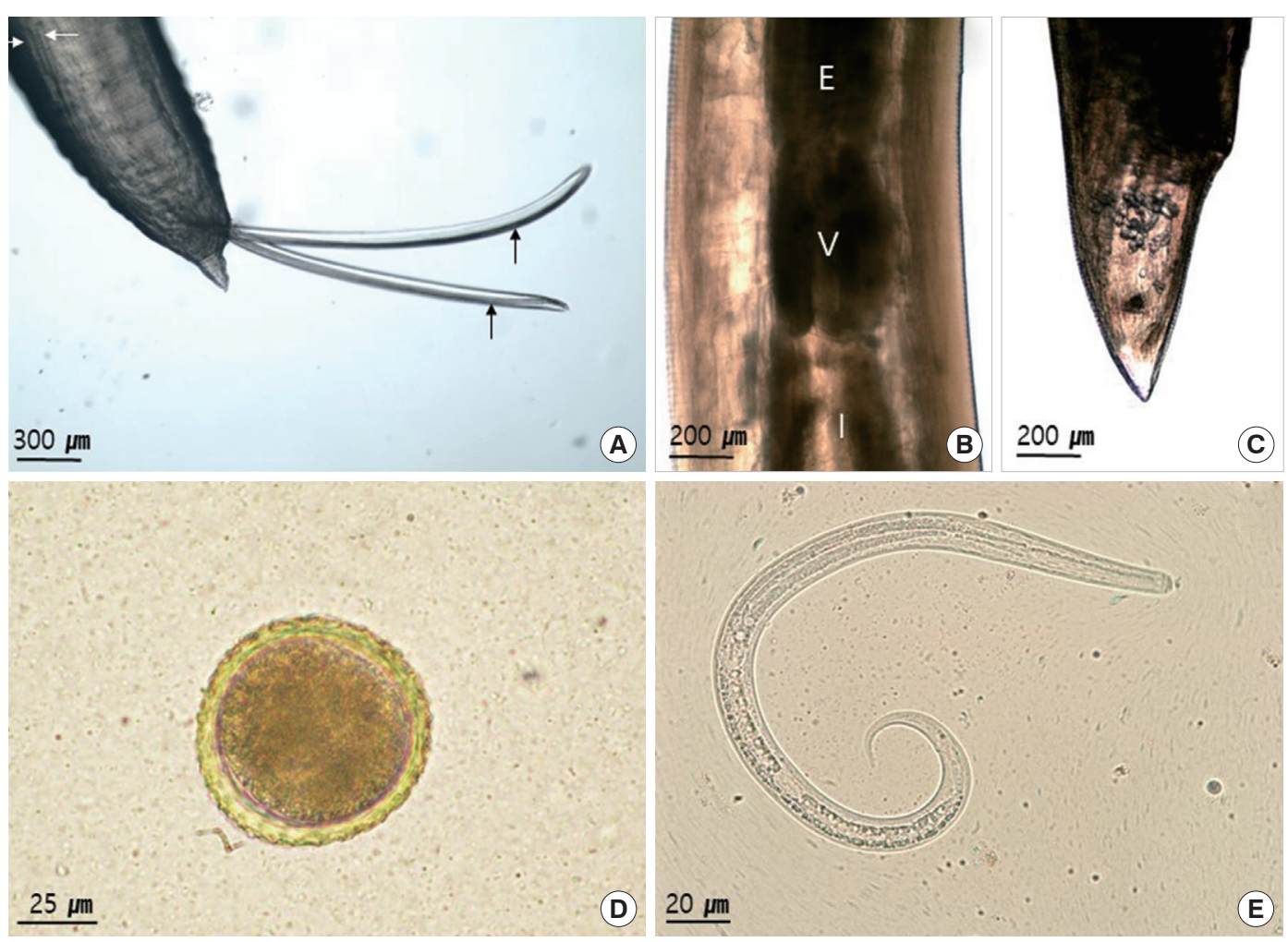

Fig. 1. Light micrographs of Toxocara tanuki. (A) Caudal end of adult male. Note the 2 separated spicules (arrows). (B) There is a ventriculus between the esophagus and the intestine. (C) Caudal end of adult female. (D) Egg from feces. Note the egg shall coated with albumin. (E) Larva from muscle. E, esophagus; I, intestine; $V$, ventriculus. 

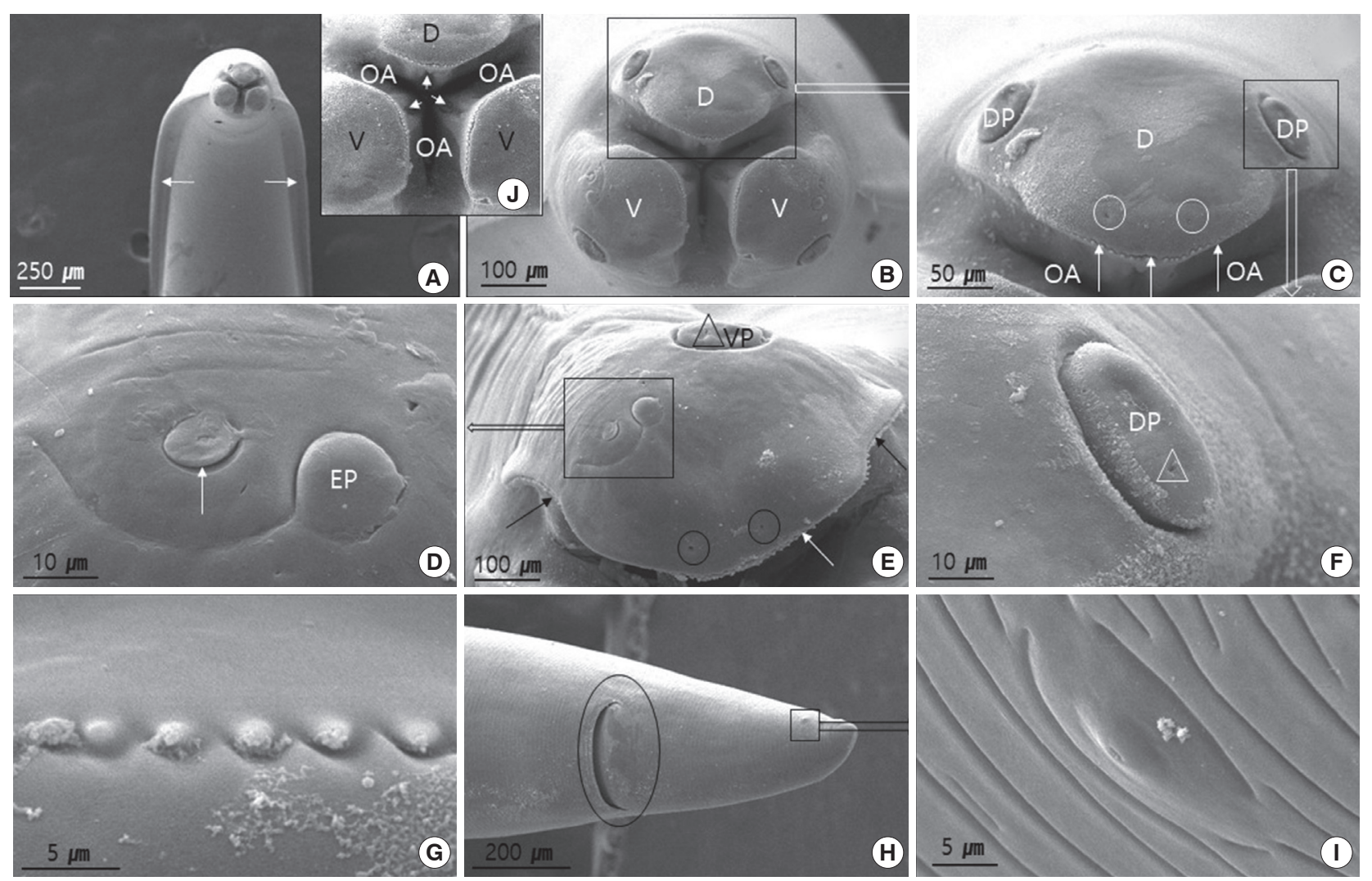

Fig. 2. Scanning electron micrographs of female Toxocara tanuki. (A) Ventral view showing anterior end. Cervical alae (arrows). (B) Frontal view showing dorsal labium, 2 ventral labium and oral aperture. (C) Dorsal labium showing a pair of big dorsolateral papillae, pit-like internal labial papillae (circles), dentigerous ridge (arrows) and oral aperture. (D) Amphid (arrow) and externo-lateral papilla on the ventral labium. (E) Ventral labium. Note the dentigerous ridge (arrows), pit-like internal labial papillae (circles), and pore (triangle) on the ventrolateral papilla. (F) Higher magnification of dorsolateral papillae on dorsal labium. Note the pore (triangle). (G) Dentigerous ridge showing unicuspid denticles. $(\mathrm{H})$ Female caudal end showing anus (circle) and phasmid (square). (I) Higher magnification of phasmid. (J) Higher magnification of triradiate oral aperture. Note the median glove (arrows). D, dorsal labium; EP, externolateral papilla; OA, oral aperture; DP, dorsolateral papilla; V, ventral labium; VP, ventrolateral papilla.

minous surface which is finely mammilated (Fig. 1D).

Fig. 2 (female) and Fig. 3 (male) show the SEM images illustrating the characteristic features of adult worm. The cuticle was finely cross-striated throughout the body. In both sexes, the anterior end of adult worm had 3 prominent labia of the ascaridoid nematodes, 1 dorsal and 2 subventral labia, surrounding the oral aperture with a median groove running longitudinally along its inner length, and the interlabia was absent (Figs. 2A, B, $\mathrm{J}$, and $3 \mathrm{~A}, \mathrm{~B})$. We also observed the triradiate oral aperture at the base of the stoma (Fig. 2B, C, J, and Fig. 3B, C). The organizations of the male and female labia were similar, except they were larger in the females. The 3 large, broad-based labia, when viewed laterally, were spade-shaped, having a deep horizontal median groove on each margin almost midway between the apex and base. Three lips have 4 relatively large papillae (2 dor- solateral papillae on the dorsal labium and 1 ventrolateral papilla on each ventral labium) (Fig. 2B, C, E, and Fig. 3B-D). Each ventral labium also had an externolateral papilla and an amphid. These 2 papillae on the ventral labium were divided and consisted of small round-to-oval, slightly raised medial elevations (amphid) and larger oval-to-round raised medial elevations (externolateral papillae) without central pores. The amphids, lateral to the externolateral papillae, appeared as small domed areas with central pores (Fig. 2D, E, and Fig. 3D). The ventrolateral papilla on the ventral labia was large and had a single pore (Fig. 2E and Fig 3D). A single row of denticles was present at the inner surface of each labium just below the anterior margin (Fig. 2C, E). Generally, denticles have a constant distribution with each other and a triangular outline. The dorsal labium, which was slightly larger than the 2 ventral labia, 

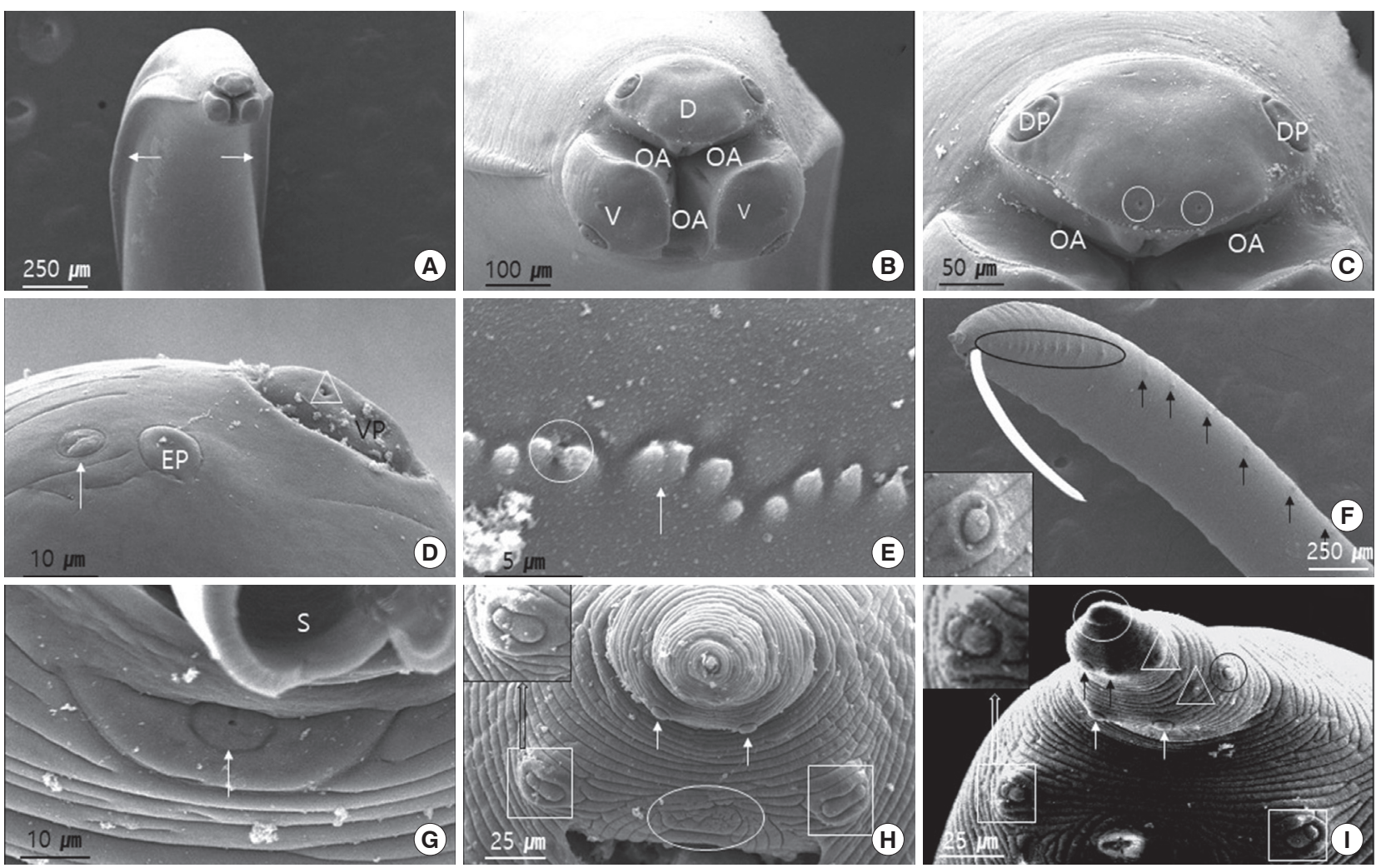

Fig. 3. Scanning electron micrographs of male Toxocara tanuki. (A) Ventral view showing anterior end. Cervical alae (arrows). Lateral crest (arrow) run longitudinally behind cervical alae (square). (B) Frontal view showing dorsal labium, ventral labium and oral aperture. (C) Dorsal labium showing a pair of big dorsolateral papillae, pit-like internal labial papillae (circles), dentigerous ridge, oral aperture. (D) Amphid (arrow) and externolateral papilla on the ventral labium. Note the pore (triangle). (E) Denticles. Note the unicuspid, bicuspid (arrow) and pits (circle). (F) Latero-ventral view. Precloacal papilla (arrows and circle). Square is a magnified precloacal papilla. (G) A small precloacal median papilla (arrow), with clear visible hole in the middle. $(H)$ Ventral view of postcloaca. A single postcloacal median papilla (circle). The first postcloacal ventral papillae (white squares). The second pair of postcloacal ventral papillae (arrows). (I) Latero-ventral view of distal end of the tail. The first postcloacal ventral papillae (white squares). A pair of second postcloacal ventral papillae (white arrows). A pair of third postcloacal ventral papillae (black arrows). Postcloacal ventro-lateral papillae (triangles). Phasmid (black circle). Mucron (white circle). D, dorsal labium; EP, externolateral papilla; OA, oral aperture; DP, dorsolateral papilla; S, spicule; V, ventral labium; VP, ventrolateral papilla.

had a range of 92-98 denticles ( $\mathrm{n}=5)$ in males and $98-104$ $(\mathrm{n}=5)$ in females. Most of the denticles were unicuspid. However, we also observed a few bicuspid denticles. We found some small round holes (pits) directly between the individual denticles in a few of the specimens (Fig. 3E). The interlabia were not present. The lateral cervical alae appeared to be narrow and long (Fig. 2A and Fig. 3A). The nematode bodies were covered with transverse striation in the cuticles. The lateral crest (indicated with an arrow in Fig. 3A) ran longitudinally behind the cervical alae. We did not observe any caudal alae. There were no morphological differences in the anterior structures of male and female. Except for the labia, the entire body displayed a fine transverse striation.

In males, the tail was relatively long, smoothly attenuated, and often had a small button-like termination (Fig. 3F, H, I).
The caudal end was ventrally curved, displaying 18 pairs of proximal precloacal papillae arranged in 2 subventral lines $(n=5)$ (Fig. 3F). The anterior edge of the cloaca had a very small precloacal median papilla, with a clear visible hole in the middle (Fig. 3G). We also observed a single postcloacal median (central) papilla (Fig. 3H). There were 5 pairs of postcloacal papillae; 3 pairs of postcloacal ventral papillae and 2 pairs of postcloacal ventro-lateral papillae (Fig. $3 \mathrm{H}, \mathrm{I}$ ) that were distributed as follows: the first pair of postcloacal ventral papillae, which was larger than the others, was observed on each side behind the cloacal opening; the other 2 pairs (2nd and 3rd postcloacal ventral papillae) were more closely associated with each other and were located near the tail end (Fig. 3H, I); the 2 pairs of postcloacal ventro-lateral papillae were posterior observed on each inner side than phasmid (Fig. 3I). The phasmid 
was located laterally between the 2nd and 3rd postcloacal ventral papillae (Fig. 3I). The distal end of the tail formed a conical appendage, similar to the rest of the body, which was transversely striated and terminated in a mucron (Fig. 3I).

In females, the tail was short, stout, and blunt, and had a large anus and no terminal mucron or caudal papillae (Fig. 1C and Fig. $2 \mathrm{H}$ ). The phasmid was approximately $1 / 4$ the distance from the tip of the tail to the anus, and slightly different in shape from that of males (Fig. 2H, I).

\section{Molecular characteristics and phylogenetic position}

One genomic DNA sample from over 100 parasite larvae, 5 samples from male adult worm and 5 samples from female adult worm were extracted and analyzed for genotyping. When ITS1 region of genomic DNA was amplified using specific primers, the PCR products indicated with a single band approximately $398 \mathrm{bp}$. After trimming about $120 \mathrm{bp}$ of the $5^{\prime}$ and 3 ' ends of the sequencing data and non-matching sequencing of samples, the $280 \mathrm{bp}$ DNA sequences were analyzed with other reference sequences.

When we analyzed with multiple alignment using ITS 1 se-

Table 2. Multiple alignment of Toxocara specimens in raccoon dogs

\begin{tabular}{lcccc}
\hline species & $\begin{array}{c}\text { Alignment } \\
\text { length (bp) }\end{array}$ & $\begin{array}{c}\text { Similarity } \\
(\%)\end{array}$ & $\begin{array}{c}\text { Variable } \\
\text { sties }\end{array}$ & Gaps \\
\hline T. tanuki & 282 & 97.2 & 1 & 7 \\
T. cati & 281 & 88.3 & 27 & 6 \\
T. canis & 282 & 87.9 & 27 & 7 \\
\hline
\end{tabular}

quence of T. tamuki, T. cati and T. canis, the sequence of the Toxocara specimens had 1 variable site and 7 gaps that differed from that of T. tanuki, and 27 variable sites that differed from that of $T$. cati or $T$. canis, respectively (Table 2). The variable sites did not match with T. cati (JF837171 or JF837173), T. canis (AJ002438, KF577855, LM051281 or LM051026) or Toxocara vitulorum (KJ365311). We defined partial ITS1 sequences of the Toxocara specimens containing identical sequences that belonged to the same clade as T. tanuki (Fig. 4). This result was also validated by the fact that they belonged to the same clades as Toxocara in the phylogenic tree based on the Neighbor-Joining method by using the ITS1 of rRNA gene. When the sequence of the Toxocara specimen was compared with genes from the nematode, the Toxocara specimen was identified as T. tanuki (AB053231).

\section{DISCUSSION}

Herein, we present a larva migrans, and a morphological and genetic description of $T$. tanuki, which parasitize Korean raccoon dogs in Korea.

Larva migrans is most common with ascaridoid nematodes. The visceral larva migrans comprises the invasion of, and migration through, any of the tissue of the animal body by nematode larvae, whether they be derived from parasites which normally mature in other host [10]. In T. canis, the life cycle is complex and according to the age of the host may involve prenatal (transuterine) and colostral (lactogenic) transmission, direct transmission or paratenic host transmission. The embryo-

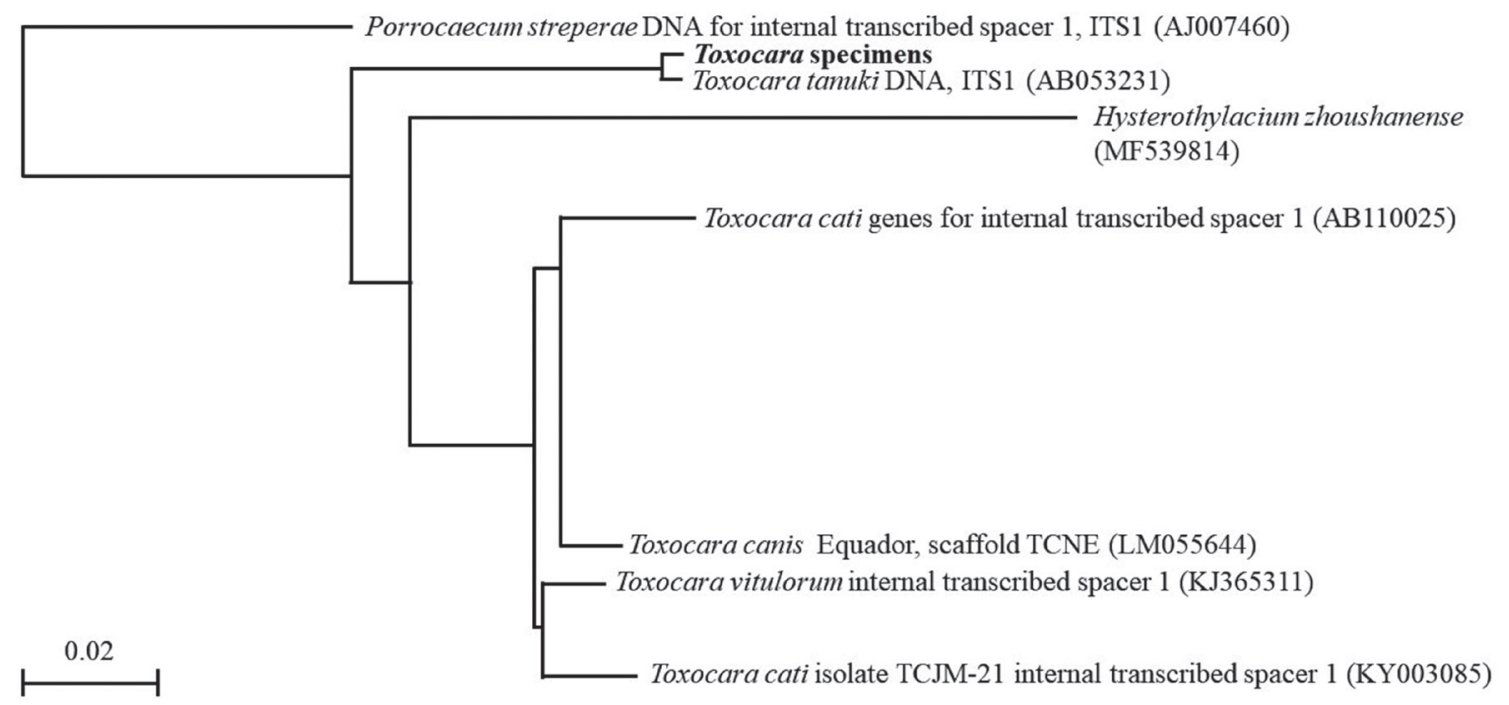

Fig. 4. Phylogenetic tree (Neighbor Joining) based on sequence analysis. 
nated eggs by ingestion are hatched in small intestine, and the larvae liberate. The larvae invade the intestinal mucosa, enter into the mesenteric and portal veins, reach the liver, and then pass to the lungs. Some larvae from the lungs pass through the bronchioles, trachea, and pharynx. And then the swallowed larvae develop into adults in the small intestine of definitive host. Other larvae, from the lungs, are migrated to the heart and are distributed throughout the internal organs (lungs, liver, kidneys, eyes, muscles, etc). Also, the larvae can infect pups through placenta. In Toxocara vitulorum, adults are found almost exclusively in calves, and prenatal and transmammary infections constitute the major sources of parasites for calves; hence the life cycle resembles that of T. canis. In T. cati, the prenatal infection does not occur, and the transmmamary infection is common [10,11]. The life cycle of T. tanuki isn't yet clear.

The larvae of T. canis, Baylisascris procyonis, Ascaris suum, and Ascaris lumbricoides showed a trend toward high survival in host muscles [12-14]. Sato et al. [5] reported an $80 \%$ incidence and an infestation intensity of 1-29 in 20 raccoon dogs that were studied in Japan. We found an incidence of 75\% and an intensity of 1-19 adults in the 20 raccoon dogs examined. Also, we found several heavily infected animals with a maximum number of 65 larvae in $50 \mathrm{~g}$ muscle sample and the larval infectivity was $75 \%$ (15/20). Therefore, we conclude that the infection and larva migrans of T. tanuki is common in the Korean raccoon dogs.

According to the characteristics of the genus Toxocara reported by Warren [15], the genus Toxocara and Toxascaris is separated from Ascaris by the arrow head, and those are separated by the egg shape. The eggs of Toxocara have corrugated shells. On the other hand, the eggs of Toxascaris have smooth egg shells. Also, the species of Toxocara that possess the cervical alae exceeding $0.19 \mathrm{~mm}$ in width include Toxocara vincenti (0.2-0.25 $\mathrm{mm}$ in male), T. canis (0.19-0.217 $\mathrm{mm}$ in both sex), T. cati (0.21-0.315 mm in both sex), and T. tanuki $(0.25 \mathrm{~mm}$ in both sex). According to the characteristics of T. tanuki reported by Yamaguti [6], the cervical alae of male are up to $0.25 \mathrm{~mm}$ wide when contracted, varying in length from 2.0 to $3.8 \mathrm{~mm}$ according to state of contraction of body and the cervical alae of female 2.3-3.0 mm long. In our study, the cervical alae measured in 2.8-3.0 mm in length and 0.18-0.2 mm in width in females, and 3.4-3.7 $\mathrm{mm}$ in length and $0.25 \mathrm{~mm}$ in width in males. In our specimens, the width of cervical alae is obviously narrower than T. cati and similar with that of T. canis.

Under a LM, the Toxocara eggs with an albuminous surface (corrugated shell) were similar in shape to those of other members of the genus Toxocara. Furthermore, measurements of egg sizes are not helpful in the differentiation of T. canis and T. cati, because approximately $90 \%$ of the eggs measured were of similar size [6]. However, the size of eggs $(71-76 \mu \mathrm{m})$ in our study was within $60-78$ by $48-63 \mu \mathrm{m}$ of $T$. tanuki by Yamaguti [6], and somewhat smaller than those of T. cati (63.7-88.1 by 53.3-73.3 $\mu \mathrm{m}$ ) by Uga et al. [16].

The characteristics of the subfamily Toxocarinae included a ventriculus without appendices and the absence of gubernaculum. Furthermore, the genus Toxocara did not have intestinal caecum and interlabia [17]. Gibbons et al. [18] reported 3 morphological identification features of Toxocara spp. (shape of cervical alae in the cross section, spicule length, and lip structure). They indicated the difference of Toxocara malaysiensis from T. canis, T. cati, and other species such as Toxocara apodemi and Toxocara mackerrasae from rodents, Toxocara paradoxu$r a$ and Toxocara sprenti from viverrids, Toxocara pteropodis from bats, T. tanuki from raccoon dog, and T. vajrasthirae from mustelids. Our specimens have a ventriculus without appendices and the absence of gubernaculum and did not have intestinal caecum and interlabia.

The diagnostic keys for Toxocara by Warren [15] indicated that the T. tanuki spicule lengths were more than $3.0 \mathrm{~mm}$ and 3.1-4.15 mm long; and were a parasite of Nyctereutes procyonoides viverrinus. The spicule sizes of our specimens $(3.0-3.5 \mathrm{~mm})$ were within 3.4-3.9 $\mathrm{mm}$ in T. tanuki by Yamaguti [6] and 3.1$4.15 \mathrm{~mm}$ by Warren [15]. The spicule length in the present specimens from $N$. p. koreensis were obviously longer than 1.705-1.902 $\mathrm{mm}$ in T. cati and $0.91-0.97 \mathrm{~mm}$ in T. canis and the others (T. pteropodis 0.58-0.725 mm, T. vincenti $0.68 \mathrm{~mm}, T$. paradoxum 2.71-3.12 mm, T. pearsei 1.2-1.32 mm) [15].

Using SEM, the arrangement of the papillae and amphids on the labia in this study agreed with the descriptions by Warren [15] and Sprent [19]; 3 lips have 4 relatively large papillae, 2 dorsolateral papillae on the dorsal labium and 1 ventrolateral papilla on each ventral labium. Each ventral labium also had an externolateral papilla and an amphid. The interlabia were not present. A row of denticles runs along the inner edge of the labia, and the number of these structures was numerous in species of ascaridoids. According to Baruš et al. [20], the average number of the denticles per labia of $T$. cati was 126, ranging from 115 to 129, T. canis 132 (122-136), and Toxascaris leonine 72 (70-75). This characteristic, according to the authors, could be used to differentiate T. cati from other species of Ascarididae. In our study, the dorsal labia display 92-98 
denticles in males and 98-104 in females. These were similar with that of T. genettae, which have 90-98 in males, and 96-102 in female [14]. These structures were lower in numbers than those of $T$. canis (122-136, mean 132) and T. cati (115-129, mean 126) [20]. However, Uni and Takada [21] suggested that the difference in the number of denticles was due to the body length rather than the difference of sex. They were arranged in a similar pattern, with digitiform and triangular denticles along the side as other ascaridoids.

Fagerholm [22] reported that the precloaca median papilla in the genus Toxocara was simple and surrounded by a cuticle of bifid appearance. Sanmartin et al. [14] indicated that the structure of the cuticle that surrounds the precloacal median papilla was highly variable in the genus Toxocara, and he did not observe the cuticle of bifid appearance in $T$. genettae. In our study, this structure was not observed to be similar as T. genettae, and was surrounded by a small cuticle crest. The precloacal median papilla with a hole was clearly located at the base that was almost at the same level as the body surface.

In male of $T$. cati, the tail has a terminal appendage, with 2025 precloacal papillae in 2 rows and 12 (6 pairs) postcloacal papillae: 1 double large pair just behind the cloaca, 2 ventral pairs, and 2 ventrolateral pair [6]. In our study, the precloacal papillae in 2 rows had 18 pairs, and a single precloacal median papilla was observed in precloacal region. Also, a single postcloacal median papilla, 3 pairs of postcloacal ventral papillae, 2 pairs of postcloacal ventro-lateral papillae, a pair of phasmid, and a mucron were observed in postcloacal region. Those are similar with $T$. cati. The postcloacal median papilla was observed in T. cati as well, but not in other species [14,21]. This papilla is a good characteristic feature of T. tanuki and T. cati, since $T$. tanuki have a smaller cranial crest in comparison with T. canis and T. leonine [15].

The presence of a single postcloacal median papilla in male T. tanuki has not been reported before, and this structure is the characteristic of T. tanuki and T. cati. The present study revealed this structure for the first time in T. tanuki from N. p. koreensis.

Determination of closely related and/or morphologically similar species is a great challenge. PCR is extremely useful in this situation, which can be used to determine unique DNA sequences for each species [23-25]. To identify parasitic specimens, several primers were used for determining the suspected species following previous reports $[26,27]$. Primer sets designed for T. canis targeted ITS1, since there are variations of this region among Toxocara spp. [28,29]. Although this primer set detected the Toxocara specimen in our results, they made multiple bands (data not shown). To resolve the non-specificity of the primer set, we redesigned the primers for $T$. tanuki, and confirmed the single band.

After Blast analysis using about $280 \mathrm{bp}$, alignment of the ITS-1 sequence of Toxocara specimen showed remarkable similarities with that of T. tanuki (AB053231), T. canis (AJ002438), T. cati (JF837171 and JF837173), and T. vitulorum (KJ365311). Furthermore, the identification was also supported by the fact that they belonged to the same clades as T. tanuki in the phylogenic tree based on the ITS1 rRNA gene. Among this sequencing data, central 183 bp of ITS1 region 100\% matched with $T$. tanuki, but only $91 \%$ matched with $T$. cati or $T$. canis. We confirmed that the ITS1 region of genomic DNA highly matched with ITS1 of T. tanuki DNA.

In conclusion, it has been found that the larva migrans and infection with T. tanuki are common in Korean raccoon dog. After confirmation of T. tanuki by DNA analysis of partial sequence arrangements of $18 \mathrm{~S}$ rRNA, we provide a thorough morphological description of $T$. tanuki characteristics based on its first SEM analysis. In morphological detail, we found that the male T. tanuki has the characteristics including specific structure for single postcloacal median papilla. Furthermore, this is the first report that Korean raccoon dogs serve as the natural definitive host of $T$. tanuki in Korea.

\section{ACKNOWLEDGMENT}

This research was supported by Basic Science Research Program through the National Research Foundation of Korea (NRF) funded by the Ministry of Education (Grant No. 2017 RIDIAIB06031728).

\section{CONFLICT OF INTEREST}

The authors declare that there are no conflicts of interest.

\section{REFERENCES}

1. Choi TY, Park CH. Home-range of raccoon dog Nyctereutes procyonoides living in the rural area of Korea. J Ecol Environ 2006; 29: 259-263 (in Korean).

2. Shin SS, Cha DJ, Cho KO, Cho HS, Choi JO, Cho SH. Arthrostoma miyazakiense (Nematoda: Ancylostomatidae) infection in raccoon dogs of Korea and experimental transmission to dogs. Korean J Parasitol 2007; 45: 121-128. 
3. Shin EH, Park JH, Guk SM, Kim JL, Chai JY. Intestinal helminth infections in feral cats and a raccoon dog on Aphaedo Island, Shinan-gun, with a special note on Gymnophalloides seoi infection in cats. Korean J Parasitol 2009; 47: 189-191.

4. Eo KY, Kwak D, Kwon OD. Detection of gastrointestinal parasites in raccoon dogs (Nyctereutes procyonoides) in the Seosan reclaimed lands, Korea. J Zoo Wildl Med 2012; 43: 682-684.

5. Sato H, Inaba T, Ihama Y, Kamiya H. Parasitological survey on wild carnivora in north-western Tohoku, Japan. J Vet Med Sci 1999; 61: 1023-1026.

6. Yamaguti S. Studies on the helminth fauna of Japan. Part 35. Mammalian nematodes, II. Jpn J Zool 1940; 21: 409-439.

7. Li F, Cui J, Wang ZQ, Jiang P. Sensitivity and optimization of artificial digestion in the inspection of meat for Trichinella spiralis. Foodborne Pathog Dis 2010; 7: 879-885.

8. Rhee JK, Seu YS, Park BK. Isolation and identification of Cryptosporidium from various animals in Korea. I. Prevalence of Cryptosporidium in various animals. Korean J Parasitol 1991; 29: 139148 (in Korean).

9. Pozio E, La Rosa G. PCR-derived methods for the identification of Trichinella parasites from animal and human samples. Methods Mol Biol 2003; 216: 299-309.

10. Sprent JFA. Visceral larva migrans. Aust J Sci 1963; 25: 344-354.

11. Soulsby EJL. Helminths, Arthropods and Protozoa of Domesticated Animals. 7th ed. London, UK. Baillière Tindall \& Cassell. 1982, pp 150-156.

12. Cho S, Egami M, Ohnuki H, Saito Y, Chinone S, Shichinohe K, Suganuma M, Akao N. Migration behaviour and pathogenesis of five ascarid nematode species in the Mongolian gerbil Meriones unguiculatus. J Helminthol 2007; 81: 43-47.

13. Sasmal NK, Acharya S, Laha R. Larval migration of Toxocara canis in piglets and transfer of larvae from infected porcine tissue to mice. J Helminthol 2008; 82: 245-249.

14. Beaver PC. Larva migrans. Exp Parasitol 1956; 5: 587-621.

15. Warren G. Studies on the morphology and taxonomy of the genera Toxocara Stiles, 1905, and Neoascaris Travassos, 1927. Zool Anz 1970; 185: 393-442.

16. Uga S, Matsuo J, Kimura D, Rai SK, Koshino Y, Igarashi K. Differentiation of Toxocara canis and T. cati eggs by light and scanning electron microscopy. Vet Parasitol 2000; 92: 287-294.

17. Anderson RC, Chabaud AG, Willmott S. CIH Keys to the Nema- tode Parasites of Vertebrates. Farnham Royal, England. Commonwealth Agricultural Bureaux. 1974, pp 1-15.

18. Gibbons LM, Jacobs DE, Sani RA. Toxocara malaysiensis N. Sp. (Nematoda: Ascaridoidea) from the Domestic Cat (Felis catus Linnaeus, 1758). J Parasitol 2001; 80: 660-665.

19. Sprent JF. The life history and development of Toxocara cati (Schrank 1788) in the domestic cat. Parasitology 1956; 46: 5478.

20. Baruš V, Wiger R, Tenora F, Staněk M. Scanning electron microscopy of the lip denticles of Toxascaris leonina, Toxocara canis and $T$. cati (Nematoda). Acta Soc Zool Bohem 1979; 43: 3-6.

21. Uni S, Takada S. The surface structure of sense organs of Toxocara cati (Schrank, 1788) viewed with the scanning electron microscope. Jpn J Parasitol 1975; 24: 365-374.

22. Fagerholm HP. Systematic implications of male caudal morphology in ascaridoid nematode parasites. Syst Parasitol 1991; 19: 215-229.

23. Hillis DM, Dixon MT. Ribosomal DNA: molecular evolution and phylogenetic inference. Q Rev Biol 1991; 66: 411-453.

24. Chilton NB, Gasser RB, Beveridge I. Differences in a ribosomal DNA sequence of morphologically indistinguishable species within the Hypodontus macropi complex (Nematoda: Strongyloidea). Int J Parasitol 1995; 25: 647-651.

25. Gasser RB, Monti JR, Bao-Zhen Q, Polderman AM, Nansen P, Chilton NB. A mutation scanning approach for the identification of hookworm species and analysis of population variation. Mol Biochem Parasitol 1998; 92: 303-312.

26. Dangoudoubiyam S, Vemulapalli R, Kazacos KR. PCR assays for detection of Baylisascaris procyonis eggs and larvae. J Parasitol 2009; 95: 571-577.

27. De Ambrogi M, Aghazadeh M, Hermosilla C, Huber D, Majnaric D, Reljic S, Elson-Riggins J. Occurrence of Baylisascaris transfu$g a$ in wild populations of European brown bears (Ursus arctos) as identified by a new PCR method. Vet Parasitol 2011; 179: 272276.

28. Jacobs DE, Zhu X, Gasser RB, Chilton NB. PCR-based methods for identification of potentially zoonotic ascaridoid parasites of the dog, fox and cat. Acta Trop 1997; 68: 191-200.

29. Zhu X, Gasser RB, Jacobs DE, Hung GC, Chilton NB. Relationships among some ascaridoid nematodes based on ribosomal DNA sequence data. Parasitol Res 2000; 86: 738-744. 
